OPEN ACCESS

Edited by:

Pedro Antonio Valdes-Sosa Centro de Neurociencias de Cuba,

Cuba

Reviewed by:

Jim Voyvodic,

Duke University, USA

Philippe CIUCIU,

Commissariat à l'Energie Atomique et aux Energies Alternatives, France

*Correspondence: Kevin J. Black kbmd@byu.net

Specialty section: This article was submitted to Brain Imaging Methods, a section of the journal Frontiers in Neuroscience

Received: 11 April 2015 Accepted: 21 March 2016 Published: 08 April 2016

Citation:

Koller JM, Vachon MJ, Bretthorst GL and Black KJ (2016) Rapid

Quantitative Pharmacodynamic Imaging with Bayesian Estimation.

Front. Neurosci. 10:144.

doi: 10.3389/fnins.2016.00144

\title{
Rapid Quantitative Pharmacodynamic Imaging with Bayesian Estimation
}

\author{
Jonathan M. Koller ${ }^{1}$, M. Jonathan Vachon ${ }^{2}$, G. Larry Bretthorst ${ }^{3}$ and Kevin J. Black ${ }^{4 *}$ \\ ${ }^{1}$ Department of Psychiatry, Washington University in St. Louis, St. Louis, MO, USA, ${ }^{2}$ College of Arts and Sciences, \\ Washington University in St. Louis, St. Louis, MO, USA, ${ }^{3}$ Department of Radiology, Washington University in St. Louis, \\ St. Louis, MO, USA, ${ }^{4}$ Departments of Psychiatry, Neurology, Radiology, and Neuroscience, Washington University in \\ St. Louis, St. Louis, MO, USA
}

We recently described rapid quantitative pharmacodynamic imaging, a novel method for estimating sensitivity of a biological system to a drug. We tested its accuracy in simulated biological signals with varying receptor sensitivity and varying levels of random noise, and presented initial proof-of-concept data from functional MRI (fMRI) studies in primate brain. However, the initial simulation testing used a simple iterative approach to estimate pharmacokinetic-pharmacodynamic (PKPD) parameters, an approach that was computationally efficient but returned parameters only from a small, discrete set of values chosen a priori. Here we revisit the simulation testing using a Bayesian method to estimate the PKPD parameters. This improved accuracy compared to our previous method, and noise without intentional signal was never interpreted as signal. We also reanalyze the $\mathrm{fMRI}$ proof-of-concept data. The success with the simulated data, and with the limited fMRI data, is a necessary first step toward further testing of rapid quantitative pharmacodynamic imaging.

Keywords: pharmacodynamics, PK-PD modeling, Bayesian parameter estimation, pharmacological imaging

\section{INTRODUCTION}

Measuring the sensitivity of an organ to a drug in vivo is a common, important research goal. The traditional approach is to independently measure biological responses to a range of different doses of drug. We recently described a novel method, rapid quantitative pharmacodynamic imaging (or QuanDyn ${ }^{\mathrm{TM}}$ ), for estimating sensitivity of a biological system to a drug in a single measurement session using repeated small doses of drug (Black et al., 2013). In that report, we tested QuanDyn ${ }^{\mathrm{TM}}$ 's accuracy in simulated data with varying receptor sensitivity and varying levels of random noise. The initial simulation testing used a simple iterative approach to estimate pharmacokinetic-pharmacodynamic (PKPD) parameters including $E C_{50}$, the plasma concentration of drug that produces half the maximum possible effect $E_{\max }$. The iterative approach was computationally efficient but could only select $E C_{50}$ from a short list of parameter values chosen a priori.

Here we revisit the simulation testing using a Bayesian method to provide continuous estimates of the PKPD parameters. The Bayesian approach also identifies data too noisy to produce meaningful parameter estimates (using a model selection package described below). Bayesian methods have been used successfully in other PKPD analyses (Lavielle, 2014, to cite but one example). For the present purpose we applied a Bayesian data analysis package specifically designed 
for efficient voxelwise analysis of 4-dimensional imaging data (Bretthorst, 2014; Bretthorst and Marutyan, 2014).

\section{METHODS}

\section{Simulated Data}

We used a standard sigmoid PKPD model (Holford and Sheiner, 1982) to create 6 time-effect curves that could reasonably represent biological signal from a pharmacological challenge study: one with no response to drug $\left(E_{\max }=0\right)$ and five with varying sensitivities to drug: $E_{\max }=10$ and $E C_{50} \in$ $\{0.25,0.6, \sqrt{2}, \pi, 7.5\}$.

As in the previous work, the concentration of drug in plasma over time is modeled as

$$
C(t)=\sum_{k=1}^{K} D_{k} \cdot u\left(t-t_{s}-t_{k}\right) \cdot 2^{-\left(t-t_{s}-t_{k}\right) / t_{1 / 2}}
$$

where $K$ doses of drug, $D_{k}$, are given at times $t_{k}, u(t)$ is the unit step function, $t_{s}$ (for "time shift") is a fixed delay between drug concentration and effect, and $t_{1 / 2}$ is the elimination half-life of drug from plasma (Black et al., 2013). Drug effect is modeled as

$$
E(C)=\frac{E_{\max } C^{n}}{\left(E C_{50}\right)^{n}+C^{n}}
$$

where $C$ is $C(t)$ from the previous equation and $n$ represents the Hill coefficient. Baseline non-quantitative signal drift was simulated by adding to each curve a quadratic function of time

$$
B(t)=a_{0}+a_{1} t+a_{2} t^{2} .
$$

The full model is then

$$
B(t)+E(C(t)) \text {. }
$$

The test curves were generated using $K=4, D_{1}=D_{2}=D_{3}=$ $D_{4}=$ the dose of drug that produces a peak plasma concentration of 1 (arbitrary concentration units), $t_{s}=0.5 \mathrm{~min}, t_{1 / 2}=41 \mathrm{~min}$, $n=1, a_{0}=1000, a_{1}=2 /(40 \mathrm{~min})$, and $a_{2}=0$. The 6 resulting curves are shown in Figure 1.

Finally we added Gaussian noise to each time point. This was done 1000 times for each of the 6 curves above and for each of 8 noise levels from $S D=0.01 E_{\text {max }}$ to $2 E_{\text {max }}$, resulting in 48,000 noisy time-signal curves plus the original 6 "clean" curves (see Supplemental Data).

\section{Testing the Method Using the Simulated Data}

In the simulated data described above, each of the 48,006 time courses were analyzed using the "Image Model Selection" package from the Bayesian Data-Analysis Toolbox (Bretthorst, 2014; Bretthorst and Marutyan, 2014). The Toolbox computes the posterior probability for the set of models (Bretthorst, 1988) given a 4d data set. A Markov chain (Gilks et al., 1996) is used to draw samples from the joint posterior probability for all of the parameters including the choice of model. The Markov chain Monte Carlo simulation included the full model $B(t)+E(C(t))$, the baseline model $B(t)$, and a "no signal" model. Each model has equal prior probability, or more precisely we specify that the conditional probability of any model, given the supplied prior probabilities for the parameters relevant to that model, is equal to that of any other model (see Bretthorst, 2014, section 22.1, at equation 22.6). Monte Carlo integration is then used to obtain samples from the posterior probability for each model and from the posterior probability for each parameter given the model. For the present analysis we specified 2500 samples at each step (50 samples run in parallel, repeated 50 times). Simulated annealing is used to minimize the risk of convergence to a nonglobal local maximum (see Bretthorst, 2014, appendix B, for details). If the posterior probability for the model

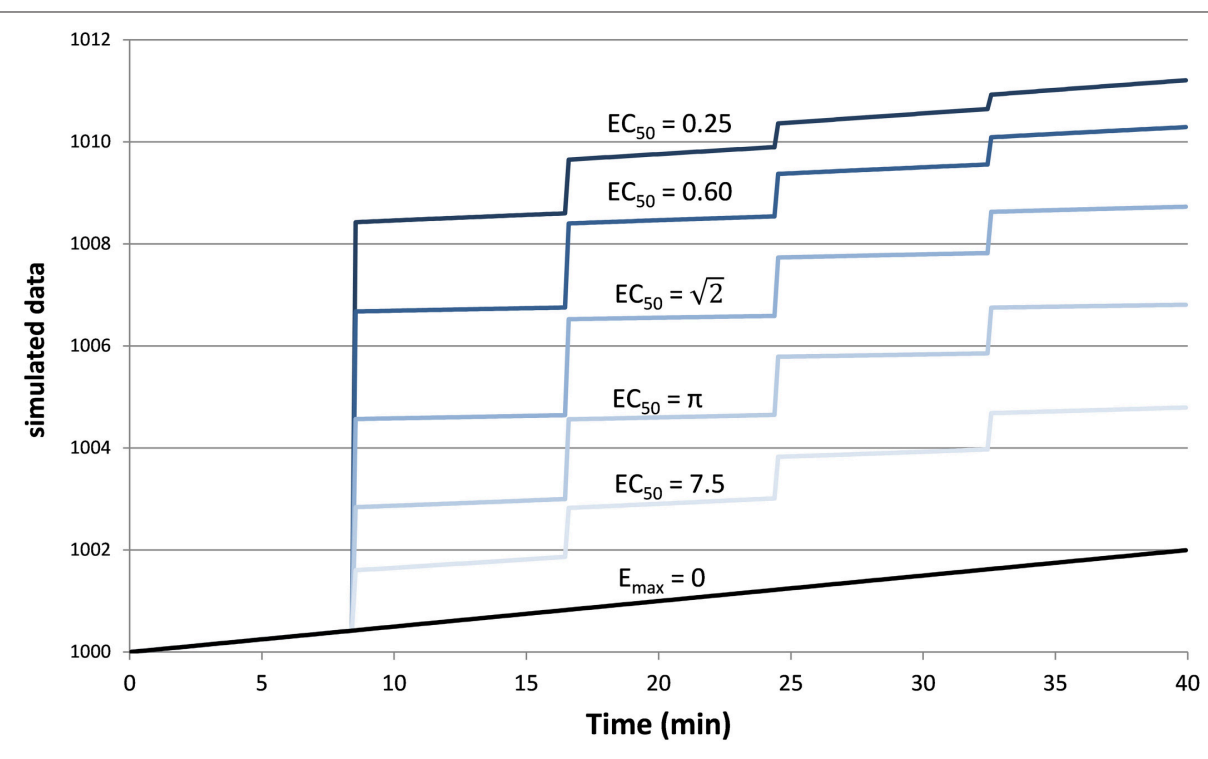

FIGURE 1 | Simulated tissue responses for various values of $E C_{50}$, i.e., the test data before adding noise. 

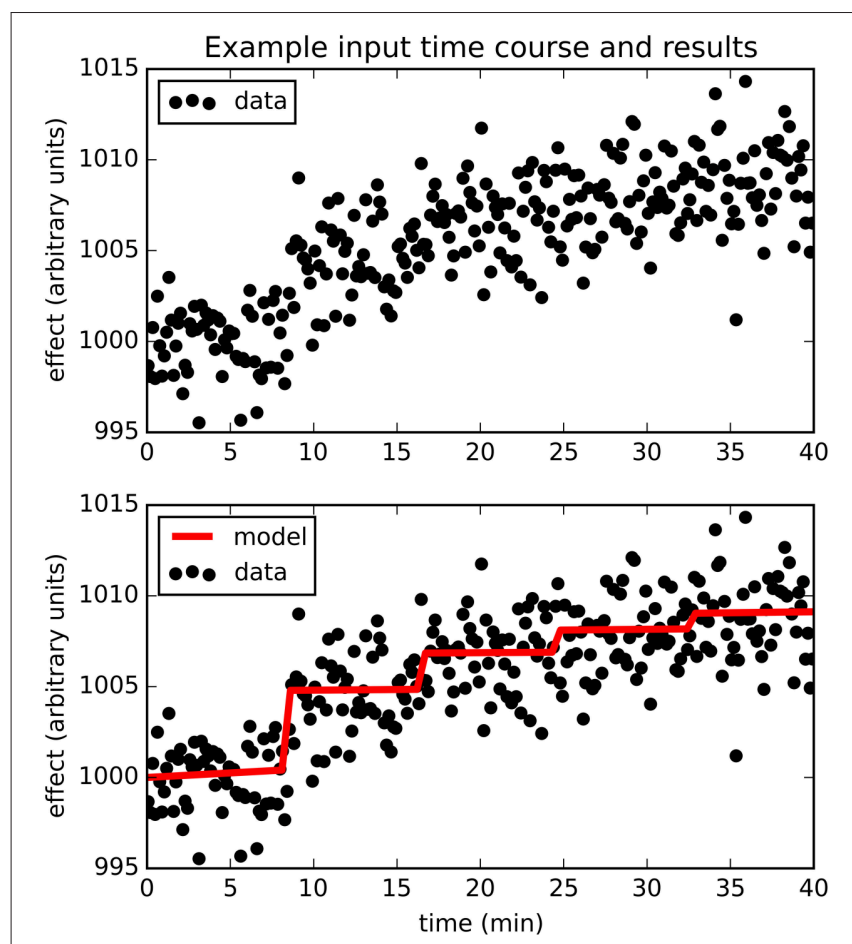

FIGURE 2 | The upper panel shows simulated dose-effect data generated using $E_{\max }=10.0, E C_{50}=\sqrt{2}, t_{s}=0.50$, added to $1000+0.05 t+0 t^{2}$ and Gaussian noise with $S D=2$. In the lower panel, superimposed on the data is the predicted time course of drug effect over time, drawn using the parameter values returned by the Bayesian Data-Analysis Toolbox as most likely given these data and the PKPD model: $E_{\max }=10.6, E C_{50}=1.43, t_{s}=0.451, a_{0}=100 \overline{0}, a_{1}=0.0553$, $a_{2}=-0.000149$. For this time course, prob(model) was estimated as 0.540, and the SD of the residuals was 2.04 .

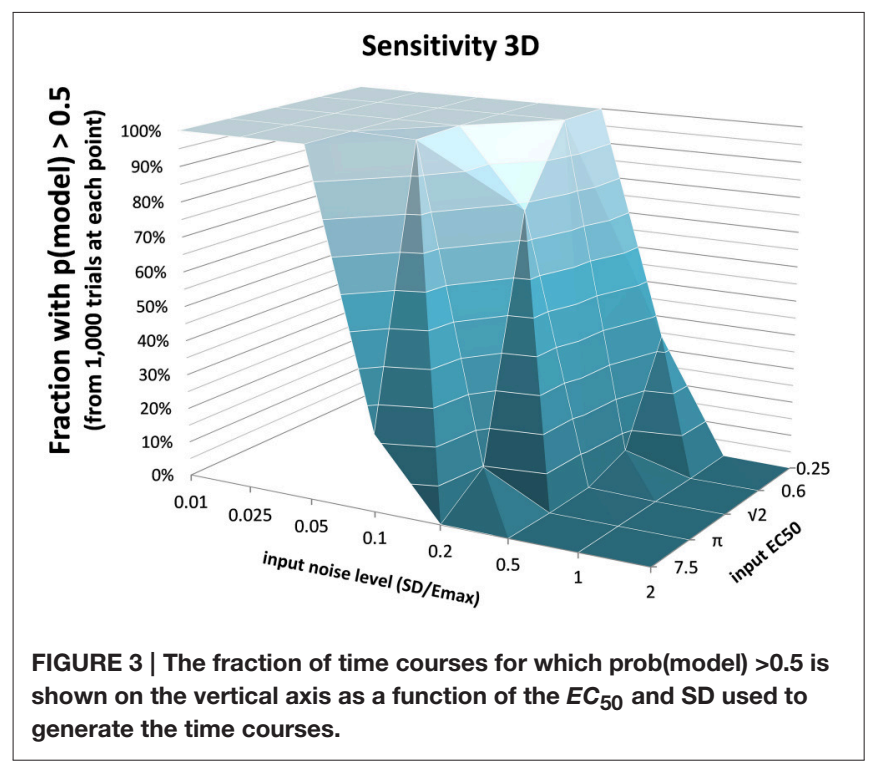

indicated the full model, $B(t)+E(C(t))$, was preferred, the package also returned values for $E C_{50}, t_{s}, E_{\max }, a_{0}, a_{1}$, and $a_{2}$. The software returns both the mean parameter values and the

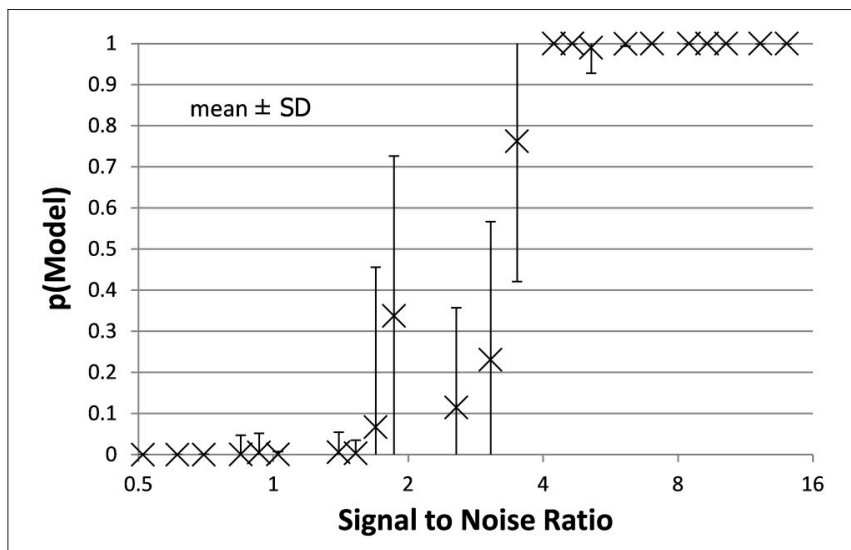

FIGURE 4 | The mean \pm SD probability of the full PKPD model is shown for each combination of $E C_{50}$ and noise as a function of that combination's SNR as defined in Section Methods. Points with SNR outside the range shown here are omitted for clarity.

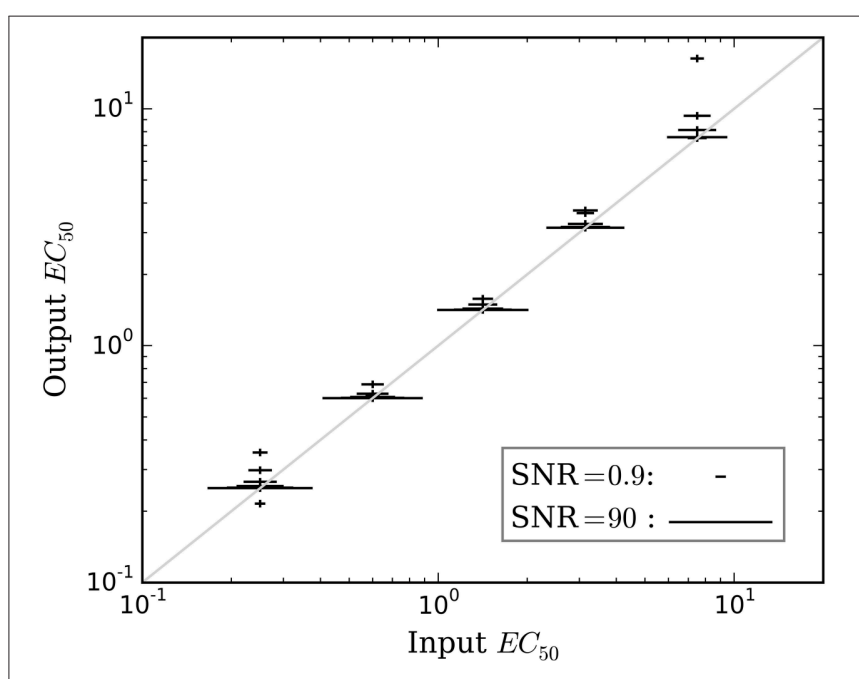

FIGURE 5 | The mean accuracy of the estimated $E C_{50}$ for time courses with prob(model) $>0.5$ is shown as a function of the input $E C_{50}$. SNR for each estimate is shown by the width of the marker, as indicated by the legend at lower right. The diagonal line indicates equality, i.e., perfect accuracy.

values from the simulation with maximum likelihood; the present report uses the latter. This analysis was repeated for each of the 48,006 time courses.

To provide more even sampling of parameter space across the conventional logarithmic abscissa for concentration-effect curves, $E C_{50}$ was coded as $10^{q}$, where $q=\log _{10} E C_{50}$, and a uniform prior probability was assumed for $q$ with range $[-3,1.3]$, corresponding to a wide range of $E C_{50}$ values from 0.001 to 20.0 . A uniform prior with range $[0,1] \mathrm{min}$ was used for the time shift parameter $t_{s}$. The Hill coefficient $n$ and the drug's elimination half-life-parameters that for biological data could be estimated separately, from a typical PK study-were fixed at $n=1$ and $t_{1 / 2}=41 \mathrm{~min} . E_{\max }$ and the coefficients of the signal drift function $a_{0}+a_{1} t+a_{2} t^{2}$ were marginalized. 


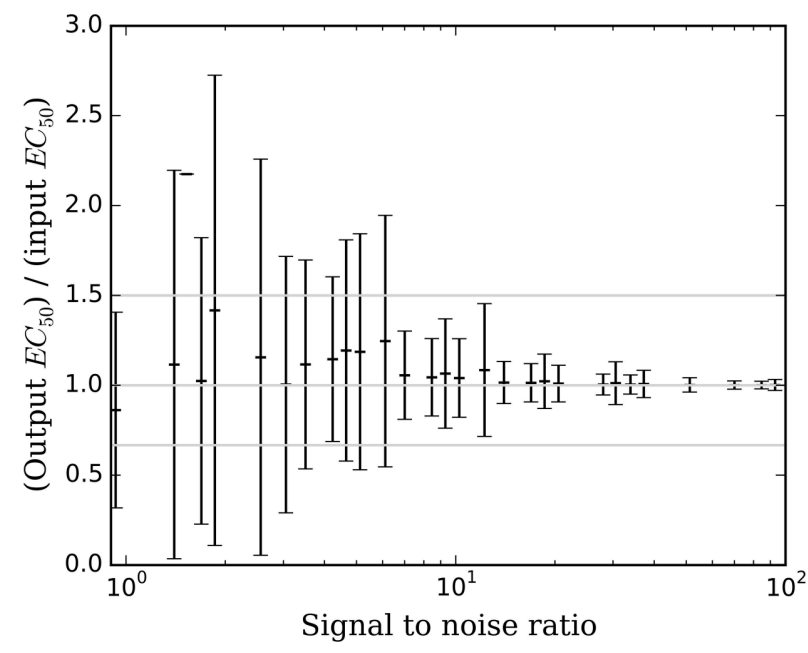

FIGURE 6 | The mean \pm SD accuracy of the estimated $E C_{50}$ for time courses with prob(model) $>0.5$ is shown as a function of SNR as defined in Section Methods. Here accuracy is defined as the output $E C_{50}$ divided by the input $E C_{50}$. The full-width horizontal lines indicate perfect accuracy (ratio $=1.0$ ) and $3 / 2$ and $2 / 3$ of perfect accuracy. The accuracy of the estimated $E C_{50}$ is superb when $S N R>$ about 6.5 , and tends to be accurate for SNR as low as 0.9.

Since tissues with high values of $E C_{50}$ respond less to a given dose of drug, i.e., $E \ll E_{\max }$, the ratio $S D / E_{\max } \ll S D / E$ underestimates the effect of noise relative to the observed effect. Therefore, we computed a signal-to-noise ratio (SNR) to simplify comparisons across the various input values of $E C_{50}$ and noise. We defined "signal" as the maximum value of $E(C(t))$, without added noise, for $0 \leq t \leq 40 \mathrm{~min}$, i.e., the local maximum of the modeled signal shortly after the last dose of drug, less the input linear drift at that same time point. In Figure 1 this value can be appreciated near the right side of the plot and ranges from about 3 for $E C_{50}=7.5$ to about 9 for $E C_{50}=0.25$. We define SNR as the ratio of this signal to the standard deviation of the added noise.

\section{Testing the Method On In vivo Data}

We tested the model described above using the same phMRI (pharmacological fMRI) data we analyzed previously with the iterative method, namely, regional BOLD-sensitive fMRI timesignal curves from midbrain and striatum in each of two animals (Black et al., 2013). These studies were approved by the Washington University Animal Studies Committee (protocols \# 20020085, 20050126). Each animal was studied twice, at least 2 weeks apart, producing 8 regional time-signal curves. On each day a total of $0.1 \mathrm{mg} / \mathrm{kg}$ of the dopamine $D_{1}$ agonist SKF82958 was given intravenously, divided into 4 equal doses on one day and into 8 equal doses on the other day (see Table 4 and Figure 10 in Black et al., 2013).

The iterative analysis had allowed only values of 5 or $30 \mathrm{~min}$ for the half-life of drug disappearance from the blood during the scan session; here we used a uniform prior probability over $[2,60] \min$ for $t_{1 / 2}$. Prior probabilities for all other parameters were the same as described above for the simulated data.
TABLE 1 | Model select results from in vivo data.

\begin{tabular}{lcclccccc}
\hline Panel & Doses & Animal & Region & Prob. & $\boldsymbol{E}_{\boldsymbol{m a x}}$ & $\boldsymbol{E C}_{\mathbf{5 0}}^{\star}$ & $\boldsymbol{t}_{\mathbf{1} / \mathbf{2}}$ & $\boldsymbol{t}_{\mathbf{s}}$ \\
\hline A & 4 & 1 & Midbrain & 1.00 & 12.59 & 3.44 & 58.33 & 0.98 \\
B & 4 & 1 & Striatum & 1.00 & -13.58 & 4.15 & 59.48 & 0.81 \\
C & 4 & 2 & Midbrain & 1.00 & 29.27 & 6.32 & 3.93 & 0.23 \\
D & 4 & 2 & Striatum & 1.00 & -2.48 & 0.001 & 40.58 & 0.01 \\
E & 8 & 1 & Midbrain & 0.00 & - & - & - & - \\
F & 8 & 1 & Striatum & 0.02 & - & - & - & - \\
G & 8 & 2 & Midbrain & 0.76 & 7.38 & 0.418 & 13.16 & 0.18 \\
H & 8 & 2 & Striatum & 1.00 & -13.9 & 1.63 & 2.00 & 0.72 \\
\hline
\end{tabular}

Panel, relevant panel in Figure 7. Prob., probability of the full PKPD model. $E C_{50}^{\star}$, the ratio of $E C_{50}$ to the peak concentration $C_{\max }$ after a single $25 \mu \mathrm{g} / \mathrm{kg}$ dose of drug. $E_{\max }$ is in BOLD signal units, and $t_{1 / 2}$ and $t_{s}$ are in min.

\section{RESULTS}

\section{Simulated Data Example}

Figure 2 provides an example result from one time course, to orient the reader to the following summary. Note that the parameter estimates are (approximately) the best estimates for the provided noisy data, even though they differ slightly from the input values used to produce the data.

\section{Sensitivity: prob(model) with Signal}

The full PKPD model explained the data better than a simpler model, i.e., prob(model) $>0.5$, except when signal was low (higher $E C_{50}$ ) or noise was substantial (Figures 3, 4).

\section{False Positives: prob(model) with Noise Only}

For the data sets containing no intentional signal, i.e., noise added to the $E_{\text {max }}=0$ line, the Toolbox never returned $p>0.5$ for any of the 8000 curves. In other words, there were no false positives.

\section{Accuracy}

Accuracy of the $E C_{50}$ estimate was considered for time courses with prob(model) $>0.5$. Figure 5 shows the mean estimated $E C_{50}$ as a function of the input $E C_{50}$; as expected, accuracy is best with higher SNR. Figure 6 shows the ratio of estimated $E C_{50}$ to input $E C_{50}$ in terms of SNR. Perfect accuracy would produce a ratio of 1.0 , and values $>1.0$ indicate overestimation of $E C_{50}$, i.e., underestimation of the sensitivity to drug.

\section{In vivo Data}

The full PKPD model was selected for 6 of the 8 regional timesignal curves (see Table 1). The data and selected model curves are shown in Figure 7.

\section{DISCUSSION}

\section{Simulation Testing}

Bayesian parameter estimation for the QuanDyn ${ }^{\mathrm{TM}}$ quantitative pharmacodynamic imaging method produced excellent results in simulated data: first, the Model Select method very accurately identified time courses with a meaningful drug-related signal, 

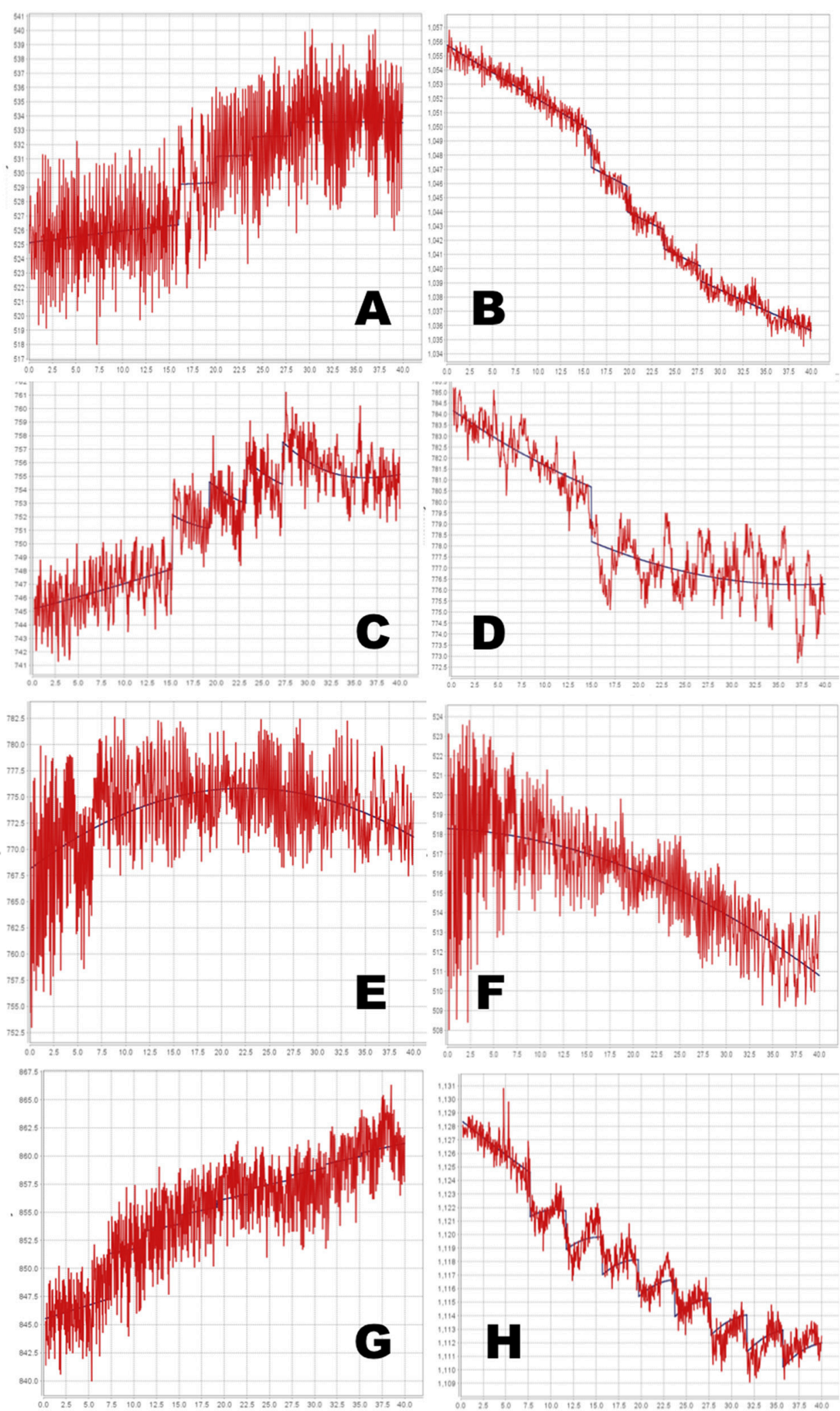

FIGURE 7 | Time-signal curves from in vivo data from a phMRI study, in red, with the selected model in dark blue. (A-D) 4-dose experiments. (E-H) 8-dose experiments. Left column, midbrain. Right column, striatum. See Table 1 for further details. 
until noise overwhelmed signal, i.e., when SNR $<$ about 3.5. The Bayesian Data-Analysis Toolbox successfully avoided false positives, correctly refraining from identifying a signal in every noise-only time course, even where sensitivity was $100 \%$. In time courses with a signal, mean accuracy was reasonable even in the face of low SNR, as shown in Figures 5, 6. Furthermore, the errors were conservative, with $E C_{50}$ usually erring on the high side (Figure 6). Said differently, the most likely quantitative error was to report slightly lower sensitivity to drug, especially when sensitivity is in fact low.

\section{Limitations}

This simulation used a simple noise model that may be best suited to a temporally stable, quantitative outcome measure, such as positron emission tomography, arterial spin labeling, or quantitative BOLD. However, because the PKPD model $E(C)$ is simply added to the baseline model $B(t)$, the latter can be replaced with a more complex signal, if needed, for non-quantitative imaging methods. For instance, Fourier series have been used to model typical BOLDsensitive fMRI data over long time intervals. The baseline model $B(t)$ could be optimized further to best suit a specific scanner, tracer or sequence, or to other experimental design choices.

Similar comments hold for the signal as well as for noise: the QuanDyn ${ }^{\mathrm{TM}}$ quantitative pharmacodynamic imaging method will perform less well if the PKPD model does not realistically model the data. However, prior to initiating an expensive imaging study, one would determine the appropriate family of PKPD models for the drug to be tested, based on traditional doseresponse experiments. We discuss this point further in Black et al. (2013). The choice of imaging method also affects the signal characteristics; for instance, typical BOLD implementations may not provide adequately linear responses to biological signal. On the other hand, using a more traditional phMRI design, the magnitude of the acute BOLD response to a single dose of drug per imaging session did increase monotonically with larger doses (Miller et al., 2013).

\section{In vivo Data}

Even with the relatively simple signal and noise models adopted for this initial testing, the tested method appeared to handle reasonably the in vivo data from a BOLD phMRI study (Figure 7). Further validation will require a larger set of similar multi-dose phMRI data, and comparison data from a more traditional dose-response study design.

The QuanDyn ${ }^{\mathrm{TM}}$ method described here has several potential advantages compared to the traditional approach to quantifying a drug effect, which is to estimate the population $E C_{50}$ by sampling a wide range of doses, one dose per subject and several subjects per dose. That approach is an excellent choice when the population under study is homogeneous (e.g., an inbred rodent strain), but does not apply well to single human subjects. One might adapt the traditional approach by repeatedly scanning a single subject, one dose per scan session, but that option brings its own complications, including scientific concerns such as sensitization or development of tolerance with repeated doses in addition to the practical and ethical consequences of repeated scanning sessions in each subject. That option, like the population method, would also require that subjects receive doses substantially higher than the $E C_{50}$, which may often be inappropriate in early human studies. Specifically, to estimate $E C_{50}$, traditional population PKPD studies require drug doses that produce effects of at least $\sim 95 \% E_{\max }$ (Dutta et al., 1996). For all these reasons, the QuanDyn ${ }^{\mathrm{TM}}$ method may prove to be a better choice when single-subject responses are important, such as for medical diagnosis or individualized treatment dosing. We elsewhere discuss potential challenges related to moving this approach into humans (Black et al., 2013).

\section{DATA DEPOSITION}

The following information was supplied regarding the deposition of related data: The simulated data sets (1000 time courses for each set of parameter values and noise level) are available at the journal web site as Supplementary Data.

\section{AUTHOR CONTRIBUTIONS}

JK performed the experiments, analyzed the data, contributed analysis tools, reviewed and critiqued the manuscript. MV performed the experiments, analyzed the data, reviewed and critiqued the manuscript. GB contributed analysis tools, reviewed and critiqued the manuscript. $\mathrm{KB}$ conceived and designed the experiments, performed the experiments, analyzed the data, wrote the paper.

\section{FUNDING}

Supported by the U.S. National Institutes of Health (NIH), grants R01 NS044598, 1 R21 MH081080-01A1, 3 R21 MH08108001A1S1, K24 MH087913 and R21 MH098670, and by the McDonnell Center for Systems Neuroscience at Washington University in St. Louis. The funders had no role in study design, data collection and analysis, decision to publish, or preparation of the manuscript.

\section{ACKNOWLEDGMENTS}

Some of these results were presented previously (JK, GB, KB: A novel analysis method for pharmacodynamic imaging. Program \#504.1, annual meeting, Society for Neuroscience, Chicago, 20 Oct 2009), and a preprint was posted on bioRxiv (DOI: 10.1101/017921).

\section{SUPPLEMENTARY MATERIAL}

The Supplementary Material for this article can be found online at: http://journal.frontiersin.org/article/10.3389/fnins. 2016.00144 


\section{REFERENCES}

Black, K. J., Koller, J. M., and Miller, B. D. (2013). Rapid quantitative pharmacodynamic imaging by a novel method: theory, simulation testing and proof of principle. PeerJ 1:e117. doi: 10.7717/ peerj. 117

Bretthorst, G. L. (1988). Bayesian Spectrum Analysis and Parameter Estimation. New York, NY: Springer-Verlag.

Bretthorst, G. L. (2014). Bayesian Data-Analysis Toolbox, Release 4.10, Manual version 2. Available online at: http://bayes.wustl.edu/Manual/BayesManual.pdf (Accessed: 04 August 2015).

Bretthorst, G. L., and Marutyan, K. (2014). Bayesian Data-Analysis Toolbox, v. 4.21 (software). Available online at: http://bayesiananalysis.wustl.edu (Accessed: 13 February 2015).

Dutta, S., Matsumoto, Y., and Ebling, W. F. (1996). Is it possible to estimate the parameters of the sigmoid $E_{\max }$ model with truncated data typical of clinical studies? J. Pharm. Sci. 85, 232-239. doi: 10.1021/ js950067y

Gilks, W. R., Richardson, S., and Spiegelhalter, D. J. (1996). Markov Chain Monte Carlo in Practice. London: Chapman \& Hall.

Holford, N. H. G., and Sheiner, L. B. (1982). Kinetics of pharmacologic response. Pharmacol. Ther. 16, 143-166. doi: 10.1016/0163-7258(82)90051-1
Lavielle, M. (2014). Mixed Effects Models for the Population Approach: Models, Tasks, Methods and Tools. Boca Raton, FL: Chapman and Hall/CRC.

Miller, B., Marks, L. A., Koller, J. M., Newman, B. J., Bretthorst, G. L., and Black, K. J. (2013). Prolactin and fMRI response to SKF38393 in the baboon. PeerJ 1:e195. doi: 10.7717/peerj.195

Conflict of Interest Statement: Authors KB and JK have intellectual property rights in the QuanDyn ${ }^{\text {TM }}$ method (U.S. Patent \#8,463,552 and patent pending 13/890,198, "Novel methods for medicinal dosage determination and diagnosis."). $\mathrm{KB}$ is an Associate Editor for the Brain Imaging Methods section of Frontiers in Neuroscience.

The other authors declare that the research was conducted in the absence of any commercial or financial relationships that could be construed as a potential conflict of interest.

Copyright (C) 2016 Koller, Vachon, Bretthorst and Black. This is an open-access article distributed under the terms of the Creative Commons Attribution License (CC BY). The use, distribution or reproduction in other forums is permitted, provided the original author(s) or licensor are credited and that the original publication in this journal is cited, in accordance with accepted academic practice. No use, distribution or reproduction is permitted which does not comply with these terms. 